\title{
Síndrome nefrótico en un recién nacido
}

\author{
Nephrotic syndrome in a newborn
}

\section{Sr. Editor:}

El síndrome nefrótico congénito (SNC) es una enfermedad poco frecuente caracterizada por la presencia de proteinuria masiva $\left(>40 \mathrm{mg} / \mathrm{m}^{2} / \mathrm{h}\right)$, hipoalbuminemia $<0,38 \mathrm{mmol} / \mathrm{L}(<2,5 \mathrm{~g} / \mathrm{dl})$, edemas e hiperlipidemia $>5,17 \mathrm{mmol} / 1$ (colesterol $>200 \mathrm{mg}$ / dl) (1), además de signos menos frecuentes como hematuria, hipertensión arterial e insuficiencia renal (2). El SNC afecta uno a tres por cada 100000 niños en el mundo (3), con mayor aparición en población finlandesa (4). Se desconoce la frecuencia de presentación de esta enfermedad en Perú.

Se presenta el caso de un neonato de 14 días de edad, nacido de parto por cesárea debido a oligohidramnios severo, prematuro (36 semanas por Capurro), con un Apgar: de 6, 8 y 9 al minuto, 5 y 10 minutos, peso al nacer de 2540 gramos, talla de $47,5 \mathrm{~cm}$ y perímetro cefálico de $33 \mathrm{~cm}$. No tenía antecedente médico familiar de importancia. El paciente presentó desde el tercer día de nacimiento oliguria e inadecuada lactancia materna con succión pobre. Al examen físico el paciente se encontró hipoactivo, reactivo a estímulos, con piel pálida, con edema $(3+/ 3+)$ a predominio de miembros inferiores. Al ingreso tenía presión arterial de 140/110 $\mathrm{mm} \mathrm{Hg}$, frecuencia cardiaca $142 x^{\prime}$, frecuencia respiratoria $56 x^{\prime}$, temperatura 36,6 ${ }^{\circ} \mathrm{C}$ y una saturación de oxígeno de $88 \%$. El resto del examen físico se encontró normal.

Los exámenes de laboratorio mostraron hemograma normal, proteína $\mathrm{C}$ reactiva cualitativa negativa, serología para virus de inmunodeficiencia humana, Treponema pallidum RPR (Rapid Plasma Reagin) y TORCH, negativas. También se encontró albúmina $1,8 \mathrm{~g} / \mathrm{dl}$, proteínas totales $3,8 \mathrm{~g} / \mathrm{dl}$, proteinuria $190 \mathrm{mg} /$ $\mathrm{m}^{2} / \mathrm{h}$, colesterol total $134 \mathrm{mg} / \mathrm{dl}$, triglicéridos $40 \mathrm{mg} /$ $\mathrm{dl}$, creatinina $2,94 \mathrm{mg} / \mathrm{dl}$, urea $81 \mathrm{mg} / \mathrm{dl}$, complemento $\mathrm{C}_{3} 0,846 \mathrm{~g} / \mathrm{l}$ y sodio $133 \mathrm{mmol} / \mathrm{l}$, calcio $0,44 \mathrm{mmol} / \mathrm{l}$ y potasio $2 \mathrm{mmol} / \mathrm{l}$. En el cultivo de orina se encontró Escherichia coli 27000 UFC/ml; además la ecografía renal mostró riñones de superficie irregular con ecogenicidad homogénea, diferenciación cortico medular ausente y diámetros renales conservados.

En cuanto al manejo, recibió $35 \mathrm{ml}$ por toma de formula láctea al $13 \%$ ocho veces al día, $3 \mathrm{ml}$ de gluconato de calcio al $10 \%$ qid, $15 \mathrm{ml}$ de albúmina humana al $20 \%$ en una hora, $3 \mathrm{mg}$ de furosemida bid, $3 \mathrm{mEq}$ de bicarbonato de sodio al 8,4\% tid, $130 \mathrm{mg}$ de ceftriaxona qd, $13 \mathrm{mg}$ de acetazolamida $\mathrm{qd}, 0,5 \mathrm{mg}$ de captopril qd y oxígeno por cánula nasal para mantener una saturación entre $88-92 \%$.

El paciente presentó un curso desfavorable por inadecuada respuesta al tratamiento establecido. Los familiares solicitaron la alta voluntaria del paciente, impidiéndose realizar un estudio anatomo-patológico y genético. Previo a la alta voluntaria se solicitó el consentimiento informado a los padres para la divulgación académica del caso.

El SNC es un cuadro clínico de presentación infrecuente con pronóstico desfavorable debido a complicaciones como sepsis y tromboembolismo

\footnotetext{
1 ASOCIEMH Cusco, Escuela Profesional de Medicina Humana, Facultad de Ciencias de la Salud, Universidad Nacional de San Antonio Abad del Cusco. Cusco, Perú.

2 Asociación de Médicos Residentes del Instituto Nacional de Salud del Niño (AMERINSN), Instituto Nacional de Salud del NiñoBreña. Lima, Perú.

Servicio de Neonatología, Hospital Nacional Adolfo Guevara Velasco, EsSalud. Cusco, Perú.

Estudiante de medicina humana;

Médico residente de Pediatría;

Médico pediatra neonatóloga.
} 
pulmonar (5). Puede ser de etiología idiopática y por mutaciones genéticas de tipo autosómico recesivo del gen NPHS1 que codifica la proteína nefrina (68), o secundario a glomerulonefritis, enfermedades sistémicas, neoplasias, entre otras (2).

En este caso no se pudo identificar la causa del cuadro clínico; sin embargo, se presume que un trastorno genético pueda ser la posible etiología al presentarse en aproximadamente el $90 \%$ de los casos de SNC en el primer trimestre de vida postnatal y por su evolución desfavorable (2). Se conoce que la mayoría de los pacientes con mutaciones del gen NPHS1 nacen prematuros y con un peso entre 1500 a 3500 gramos, como el caso que reportamos (7); no descartándose el síndrome de Pierson por la rapidez de instauración de la insuficiencia renal (2).

El diagnóstico de este síndrome podría sospecharse desde la etapa prenatal por la elevada presencia de marcadores como la alfafetoproteína (AFP) en suero materno y valores normales de colinesterasa a partir de la semana 15 de gestación $(5,6)$ y ante la evidencia de riñones grandes e hiperecogénicos en la ultrasonografía (6). Posterior al nacimiento, se sospecha ante un caso de prematuridad y de placenta grande (5); realizándose el diagnóstico por presencia de manifestaciones clínicas tales como edema, proteinuria e hipoalbuminemia $(1,2)$ y por estudio genético, previo descarte de causas secundarias con el propósito de poder determinar la etiología específica y establecer el manejo terapéutico y profiláctico más adecuado $(2,8,9)$. El tratamiento tiene como objetivo controlar la proteinuria, recuperar la diuresis, reestablecer los niveles de albúmina y prevenir las complicaciones, mediante una buena nutrición, el uso de diuréticos, la administración de albúmina humana, la profilaxis con antibióticos, entre otras $(2,9)$. Una respuesta positiva al uso de corticoides es favorable en el pronóstico del paciente $(2,10)$. No obstante, el trasplante renal es la terapia que mejor calidad de vida puede otorgar al paciente (5).

\footnotetext{
Maycol Suker Ccorahua-Rios ${ }^{1,}$, Noé AtamariAnahui ${ }^{2, b}$, Berioska Lezama-Ferro ${ }^{1,}$, Rodrigo Quispe-Curo ${ }^{1, a}$, Valeria Béjar-Yabar ${ }^{1,}$, , Gloria Janeth Yabar-Galdos ${ }^{3, c}$
}

\section{Correspondencia:}

Maycol Suker Ccorahua-Rios

Av. Uruguay M-3, Cusco, Perú

Teléfono: (51) 964217232

Correo electrónico: maycol.s.c.r@hotmail.com

\section{REFERENCIAS BIBLIOGRÁFICAS}

1. León DC, Agudelo AM, Ramos J, Ibarra MD. Caracterización clínica del síndrome nefrótico en infantes de Neiva. Rev Fac Salud. 2016;7(1):9-16.

2. Román E. Síndrome nefrótico pediátrico. Protoc diagn ter pediatr. 2014; 1:283-301.

3. Genetics Home Reference. Congenital nephrotic syndrome. Washington DC: Genet Home Ref; 2016. (Fecha de acceso 23 de diciembre de 2018) Disponible en: https://ghr.nlm.nih.gov/condition/congenitalnephrotic-syndrome

4. Kumoji R, Badoe E. Congenital nephrotic syndrome of the Finnish type. Ghana Med J. 2008; 42(1):42-4.

5. Canalejo González D, González Rodríguez JD, Navas López VM, Sanchez-Moreno A, López-Viota JF, Martín-Govantes J. Evaluación de las estrategias terapéuticas en el síndrome nefrótico congénito tipo finlandés. An Pediatr. 2006; 65(6):561-8.

6. Cobos-Carrascosa E, Campos-Aguilera A, DazaTorres A. Multigene involvement in congenital nephrotic syndrome. Nefrología. 2014; 34(2):268-70.

7. Jalanko H, Holmberg C. Congenital nephrotic syndrome. En: Avne E, Harmon W, Niaudet P, Yoshikawa N, Emma F, Goldstein S, editores. Pediatric Nephrology. Finlandia: Springer; 2014. p. $1-29$.

8. Yoshizawa C, Kobayashi Y, Ikeuchi Y, Tashiro M, Kakegawa S, Watanabe T, et al. Congenital nephrotic syndrome with a novel NPHS1 mutation. Pediatr Int. 2016; 58(11):1211-5.

9. Lasso M, Adragna M, Ayub I, Ghezzi L, Sierra A, Zalba J. Consenso de tratamiento del síndrome nefrótico en la infancia. Arch Argent Pediatr. 2014; 112(3):277-84.

10. Durán Álvarez S. De lo simple a lo complejo en el síndrome nefrótico. Rev Cubana Pediatr. 2014; 86(2):129-33. 